\title{
La santé à la télévision : émergence d'une question sociale
}

Health on television: emergence of a social issue

\section{Hélène Romeyer}

\section{(2) OpenEdition}

\section{Journals}

Édition électronique

URL : http://journals.openedition.org/questionsdecommunication/7328

DOI : 10.4000/questionsdecommunication.7328

ISSN : 2259-8901

Éditeur

Presses universitaires de Lorraine

\section{Édition imprimée}

Date de publication : 1 juillet 2007

Pagination : $51-70$

ISBN : 978-2-86480-829-9

ISSN : 1633-5961

Référence électronique

Hélène Romeyer, « La santé à la télévision : émergence d'une question sociale », Questions de communication [En ligne], 11 | 2007, mis en ligne le 01 juillet 2007, consulté le 12 avril 2021. URL: http://journals.openedition.org/questionsdecommunication/7328; DOI : https://doi.org/10.4000/ questionsdecommunication.7328 


\section{$>$ DOSSIER}

HÉLÈNE ROMEYER

IUT Lannion, université Rennes 1

Groupe de recherche sur les enjeux de la communication

Université Grenoble 3

helene.romeyer@u-grenoble3.fr

\section{LA SANTÉ À LA TÉLÉVISION : ÉMERGENCE D'UNE QUESTION SOCIALE}

Résumé. - La place de la santé à la télévision française a évolué. Un temps cantonnée aux émissions spécialisées, la thématique a lentement irradié les émissions de société, puis toute la grille de programmation. La santé a ensuite quitté la sphère scientifique et médicale, ce qui est analysé ici à travers l'utilisation et le rôle des témoignages. Ces mutations sont révélatrices de deux phénomènes : d'une part, des enjeux socioprofessionnels tant pour les médecins que pour les journalistes, mais aussi pour les hommes politiques, les malades et les associations; d'autre part, du phénomène de « socialisation » de la maladie notamment sous l'action des médias de masse.

Mots clés. - Santé, télévision, témoignage, espace public, logique sociale, cancer. 
$\grave{A}$ la suite de plusieurs affaires (sang contaminé, hépatite $B$, amiante,...) et, surtout, avec la médiatisation de la lutte contre le sida, les modalités du débat sur la santé et sur la médecine ont évolué : les malades et les associations sont devenus des acteurs privilégiés des émissions de télévision, effaçant peu à peu la place des professionnels de santé et des professionnels de l'information (Marchetti, 1997 ; Henry, 2000 ; Barbot, 2002 ; Dodier, 2003)'. Médecine et santé débordent alors du seul cadre des émissions médicales et donc de l'unique sphère scientifique pour intégrer celle des émissions de société (Soulages, 2003 : I). Si nous nous intéressons ici à l'évolution actuelle des émissions sur la santé, à travers la thématique du cancer, c'est parce que celles-ci sont révélatrices à la fois d'évolutions sociales et de l'évolution structurelle de la télévision.

En effet, la télévision est intrinsèquement liée à des évolutions sociétales larges qui rendent un discours dicible et recevable à un moment donné, en fonction de mutations sociohistoriques profondes, et sur le long terme. Ainsi le cancer est-il présent dans la mémoire et les représentations collectives, d'une part, parce que la maladie est inhérente à l'existence et, d'autre part, parce qu'elle touche ou a touché presque toutes les familles de près ou de loin. Contrairement à d'autres maladies ayant bénéficié d'une exposition médiatique comme le sida ou le sang contaminé, le cancer ne connaît pas d'événements déclencheurs, pas d'urgence sanitaire ni politique, pas de scandales politico-sanitaires, pas de population ou comportements à risque, et donc aucun phénomène « d'emballement médiatique ». Ainsi une première étude quantitative sur la base du dépôt légal de l'INA permet-elle d'établir que la médiatisation du cancer existe depuis longtemps et sous une forme bien établie. Excepté un pic en 1996, qui correspond conjointement au scandale de l'Association pour la recherche sur le cancer (ARC), à la mort de François Mitterrand, au livre du médecin de celui-ci, Claude

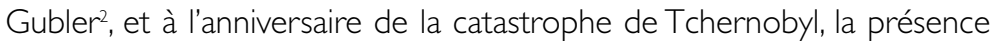
du cancer ne laisse apparaitre aucune fluctuation quantitative flagrante. Toutefois, depuis 2003, la présence des malades tend à s'équilibrer avec

\footnotetext{
' Ce texte s'appuie sur une étude menée dans le cadre d'un post-doctorat au sein de I'Institut national du cancer, département Sciences humaines, d'octobre 2005 à septembre 2006.

${ }^{2}$ Avec le journaliste M. Gonod, Cl. Gubler a publié Le grand secret (Paris, Plon, 1996) dans lequel il raconte comment, en tant que médecin personnel de Fr. Mitterrand, il signait sur ordre du président, tous les semestres, un bulletin de santé ne mentionnant pas ses problèmes de santé. Le livre fut interdit à la vente après que l'auteur et la maison d'édition aient été condamnés pour violation du secret médical par la famille de Fr. Mitterrand. Le 18 mai 2004, la Cour européenne des droits de l'homme a ensuite condamné la France pour avoir interdit définitivement Le Grand secret. Suite à ceci, celuici fut réédité aux Éditions du Rocher (Monaco/Paris, en 2005).
} 
celle des médecins et, les journalistes ne sont plus des journalistes spécialisés. C'est pourquoi nous proposons ici une analyse qualitative à partir d'un corpus réduit (2000-2005). Le choix des bornes chronologiques repose sur l'hypothèse que si le discours de Jacques Chirac le 14 juillet 2002, n'avait pas provoqué d'évolution quantitative, le statut du porteur du message et l'instauration d'un chantier présidentiel - donc d'une forme de priorité - avaient provoqué une mutation qualitative des discours sur le cancer.

Du point de vue de l'évolution structurelle, et jusqu'à la fin des années 80, deux types de représentations de la santé coexistent à la télévision : des émissions spécialisées sur la santé et la recherche médicale ${ }^{3}$, et des émissions de société qui abordent la santé, comme d'autres thématiques, par son aspect compassionnel, à travers des témoignages et des reportages (Delforce, 2004 ; de Cheveigné in : Pailliart, 2005). Les unes ont pour objectif de diffuser des informations scientifiques, les autres se saisissent d'une thématique de santé pour la mettre en scène et susciter de l'empathie à travers la présentation de récits de vie et de discussions en plateau. Depuis, un troisième type d'émission - les émissions de santé - s'est imposé par un glissement mutuel des émissions médicales vers les émissions de société. Cette évolution est due à une mutation structurelle du média lui-même et non à la thématique du cancer, celle de l'émergence des témoignages renvoyant à la télévision de l'intimité (Mehl, 1996), celle de la promotion de la parole ordinaire en lieu et place de l'expert et parfois même du journaliste (Cardon, Heurtin, 1999 ; Romeyer, 2004). Cette tendance, plusieurs fois étudiée, est particulièrement adaptée à la thématique santé où il s'agit de faire part de son expérience sur un mode éminemment émotionnel, d'exposer sa souffrance, et d'évoquer publiquement son intimité.

Alors comment saisir en même temps et sans les opposer, les mutations liées à l'évolution sociale, celles liées à l'évolution structurelle des médias, et celles spécifiques à la télévision, sur un sujet précis comme celui des formes de mise en visibilité du cancer à la télévision ? Si les différentes approches de la télévision restent souvent spécialisées (linguistique, sémiologique, économique, sociologique, historique), nous prônerons une analyse du média dans son ensemble, c'est-à-dire à la fois comme une entreprise de production appelant une certaine rentabilité, comme un programme culturel et de divertissement, et comme un outil d'information. II s'agit donc de dépasser l'analyse des faits linguistiques ou celle des images, de dépasser les analyses sur les effets supposés de la

\footnotetext{
${ }^{3}$ Nous parlerons ici d'émissions médicales pour évoquer les émissions spécialisées dans la recherche et l'information médicales. II s'agit par exemple de Savoir plus santé, du Magazine de la santé, ou bien encore des reportages médicaux dans les journaux télévisés.
} 
télévision, pour envisager les différentes formes de discours de la télévision, discours entendu dans son acception la plus large, à savoir non limité à sa dimension linguistique. Selon nous, c'est la meilleure façon d'envisager le caractère protéiforme de la télévision et les multiples enjeux que revêt l'évolution de la santé à la télévision. Ainsi inscrivonsnous notre démarche dans une recherche de « mouvements de longue durée portant aussi bien sur des processus de production que sur des processus de consommation ou des mécanismes de formation des usages » (Miège, 1989 : 18-19), c'est-à-dire dans une volonté de faire émerger des « logiques sociales ». Ce faisant, notre objectif permet de dépasser l'opposition entre approche macro et micro, entre phénomènes structurels et phénomènes langagiers. Plus précisément, au niveau macro, l'ancrage social et politique du cancer rend possible le discours médiatique sur la maladie et les malades, ainsi que sur les évolutions sociétales qui en font évoluer les modalités. Ceci se vérifie au niveau micro par plusieurs mutations particulières comme le rôle, l'évolution et la place du témoignage des malades. Enfin, les enjeux de ces mutations se situent au niveau méso, là où s'articulent les stratégies d'acteurs, les enjeux identitaires à la fois des médecins, des journalistes, des journalistes spécialisés, des associations, etc.

À travers l'analyse des émissions médicales et généralistes ayant abordé le cancer entre 2000 et 2005, nous évoquerons d'abord la permanence de deux formes de mise en visibilité de la santé : émissions spécialisées et émissions de société abordant la santé. Si le genre « spécialisé » perdure, nous verrons comment, par une intégration des normes de fonctionnement des émissions de société - notamment les témoignages -, il s'est vidé de sa substance. II n'est plus alors qu'une étiquette permettant de nommer et classer un produit dans une grille de programmation. Enfin, en devenant un problème social, la santé semble quitter la sphère scientifique et médicale, et provoquer l'émergence d'un nouveau type d'émission : les émissions de santé. Ce glissement soustendu par des enjeux importants n'est pas sans provoquer quelques réactions. À l'aune des positionnements théoriques et méthodologiques décrits, l'analyse du discours télévisuel - tel que défini supra - met ainsi en évidence l'émergence d'un nouveau type d'émissions, révélatrices à la fois d'évolutions sociétales et d'évolutions structurelles du média.

\section{L'espace télévisuel traditionnel de la médecine}

L'allongement de la scolarité, la multiplication des hebdomadaires généralistes, l'attention croissante portée aux médias, la diffusion d'informations sur l'internet ont contribué à créer un volume important de connaissances médicales accessibles. Actuellement, un utilisateur averti des médias peut se tenir informé des récentes avancées thérapeutiques 
aussi bien qu'un médecin. Le généraliste, s'il garde l'avantage de l'expérience et de l'accès aux circuits hospitaliers, tend à perdre cette singularité qui le posait en « homme de l'art » face à son patient.

Une enquête réalisée par le CSA en 2000, et réactualisée en 2002, établit que les émissions scientifiques sont moins nombreuses que les émissions de divertissement; et parmi les programmes scientifiques, les sujets médicaux sont relativement moins nombreux que ceux de sciences humaines (les reportages à caractères historiques et archéologiques chaque semaine sur Arte ou encore l'Odyssée de l'espèce, par exemple). Relativement, car l'étude a montré qu'entre 1990 et 2002, l'offre globale d'émissions médicales (Le Journal de la Santé, le Magazine de la santé, Savoir plus santé, les émissions spéciales sur le sida ou les maladies génétiques) a été multipliée par 4,4 contre 3,5 pour les émissions abordant des sujets de sciences dites exactes (Savoir plus sciences, E=M6, Rayons $X$ ). Les émissions médicales sont donc plus présentes sur les écrans et les thématiques de santé largement plébiscitées, ce qui contribue à leur construire un domaine de plus en plus autonome parmi l'offre de programmes télévisés. En effet, traditionnellement, les émissions touchant au corps humain bénéficient d'une bonne audience : Savoir plus santét, programmé à une heure relativement peu favorable, le samedi en début d'après-midi sur France 2, rassemble plus de 3 millions de téléspectateurs ${ }^{5}$. Le Magazine de la santét sur France 5 connaît une moindre audience, mais c'est une émission quotidienne. Depuis deux décennies, la santé a surtout gagné les émissions de société : Ça se discute, C'est au programme, Zone interdite, etc. Ce sont ainsi deux univers qui se côtoient à la télévision avec un partage des rôles bien installé.

\footnotetext{
${ }^{4}$ Savoir plus santé est une émission hebdomadaire diffusée sur France 2, le samedi à I 3 h 45 depuis 1994. Elle est présentée par S. Aurenche, journaliste, et L. Broomhead, animateur. À noter que cette émission est devenue en septembre 2006, Sentez-vous bien, transposant jusque dans son titre l'évolution des émissions dites spécialisées. France 2 a par ailleurs décidé en mars 2007 d'en arrêter la diffusion, ce qui supprime le genre « émission scientifique » de la grille de programmation de France 2.

${ }^{5}$ Données Médiamétrie.

${ }^{6}$ Le Magazine de la santé, émission quotidienne sur France 5, du lundi au vendredi à I3h 40, présentée par M. Cymes, médecin et chroniqueur médical, et M. Carrère d'Encausse, journaliste.

7 Journaliste, P. Desgraupes a marqué la télévision française de son empreinte pendant près d'un demi siècle. II créé notamment en 1959 la première grande émission d'informations et de reportages avec Cinq colonnes à la Une. II a été successivement directeur de l'information sur la première chaîne de 1968 à 197I, nommé par J. Chaban-Delmas, puis directeur général d'Antenne 2 en 1981 .
} 


\section{La santé dans les émissions médicales : une affaire de spécialistes}

Ainsi, pour fournir des informations sur la santé, les émissions médicales ont-elles toujours fait appel à des spécialistes ou du moins à des présentateurs investis d'une mission pédagogique : Pierre Desgraupes ${ }^{7}$, le regard vissé à la caméra annonce : «Nous allons tout vous expliquer sur le fonctionnement de l'intelligence ». La formule magistrale des années 60 résiste au temps tout comme le ton didactique auquel aucun des successeurs de ce pionnier n'a renoncé. Par exemple, Le Magazine de la santé du 17 janvier 2004 propose cet échange composé de propos explicatifs et de schémas ${ }^{8}$ :

- I' 49 Marina Carrère d'Encausse : « II n'existe pas un seul type de cancer du sein et pour les distinguer il faut d'abord savoir comment est fait un sein. Le sein est fait de plusieurs composants $\gg$.

I' 56 Coupe transversale d'un sein et les explications de son fonctionnement.

- 2' 09" Marina Carrère d'Encausse : " Et parmi les grands types de cancer qui existent la grande majorité se développe à partir des cellules dans ces canaux » (elle montre sur le schéma).

2' 20" Michel Cymes: « Alors des origines différentes mais tous ces types de cancer peuvent revêtir des gravités différentes aussi parce que les cellules cancéreuses peuvent rester dans ces canaux... » (il pointe les canaux sur le schéma).

Parfois, le programme télévisé montre les sondes, les perfusions, le sang, la réalité des soins, comme cette tumeur du sein exposée à la caméra, dans le Magazine de la Santé du 17 janvier 2004. De même, de nombreuses images de matériel médical illustrent ces émissions. Ces images sont rarement commentées, elles semblent se suffire à ellesmêmes : leur rôle est de garantir le sérieux et le caractère scientifique de la médecine. Sophie Aurenche, présentatrice de Savoir plus santé déclare d'ailleurs que « la force de la télévision est de montrer et donc, une image d'un bloc opératoire, ou un schéma en 3 dimensions sont bien plus explicatifs que de longs discours $\gg^{9}$. Même succincte, une figuration animée est préférable à un cliché photographique. Pour suggérer un processus évolutif, le texte appuyé sur des dessins distingue des phases, énumère et représente successivement des phénomènes simultanés. Ces programmes médicaux soulignent les avancées de la recherche et développent un discours qui se veut vulgarisateur. Celui-ci concerne à la fois les émissions médicales (Savoir plus santé, Le Magazine de la santé) et

\footnotetext{
${ }^{8}$ Pour chaque extrait d'émission dans ce document, nous donnons les time code des propos cités, time code remis à zéro pour chaque début d'émission, indépendamment de I'heure de programmation.

${ }^{9}$ S. Aurenche, journaliste, co-présentatrice de l'émission Savoir plus santé sur France 2 depuis 2004 (entretien réalisé le 30 mars 2006).
} 
les journaux télévisés. Ainsi Jean-Daniel Flaysakier ${ }^{10}$ déclare-t-il : « Mon rôle est d'expliquer des choses très compliquées, en étant le plus simple possible, et le tout dans un climat de pression car ce sont toujours des sujets d'actualité donc d'inquiétude. Et je dois le faire en très peu de temps à l'intérieur du journal télévisé ». Les acteurs principaux de ces programmes sont donc des journalistes spécialisés comme Sophie Aurenche, des journalistes-médecins tels Michel Cymes ou scientifique comme Jean-Daniel Flaysakier, ainsi que des médecins ou personnels médicaux et des scientifiques. Pour appuyer leurs propos et mettre en valeur l'image de la science, les magazines médicaux proposent des reportages scientifiques où de multiples spécialistes sont interviewés sur leur lieu de travail avec toujours un bandeau de présentation en bas de l'écran qui rappelle leur appartenance aux professions médicales". C'est donc une volonté d'encadrement didactique des sciences qui accompagne ces émissions avec de nombreux schémas, des images en trois dimensions, etc. Autant de caractéristiques traditionnelles renvoyant à la mission d'information de la télévision qui cohabitent avec un autre univers, plus contemporain de la télévision mettant en scène témoignage et intimité.

\section{Les magazines de société et la santé : des émissions de service}

En effet, profondément intégré depuis au moins quatre décennies à l'univers familial, le téléviseur se fond dans le décor quotidien et en partage la banalité. Une large gamme de programmes a été conçue pour renforcer cette banalisation. Tel est le cas des émissions qui développent des services pratiques sur des thématiques de santé et non des explications. Ainsi E=M6 ou Cest au programme abordent-elles des questions simples et sous un aspect généralement pragmatique. La médecine tient une large place dans ce genre d'émissions. Même Savoir plus santé, sans doute pour dédramatiser son sujet, se construit à partir de problèmes précis auxquels sont apportés des réponses brèves et claires. Ces émissions de service s'apparentent à ces magazines de presse écrite de conseils (Santé magazine par exemple) qu'on achète parce qu'ils fournissent des recommandations utiles. C'est ce qu'Éric Giacometti appelle «faire de la bobologie », expression qu'il utilise pour qualifier les émissions de santé à la télévision ${ }^{12}$.

\footnotetext{
${ }^{10} \mathrm{~J}$.-D. Flaysakier, scientifique, chroniqueur médical des journaux télévisés de France 2 (entretien réalisé le 23 mai 2006).

" « On insiste sur la légitimité des scientifiques avec les bandeaux de présentation [...] ils sont là "au titre de" », S. Aurenche (entretien réalisé le 30 mars 2006).

12 É. Giacometti est journaliste au Parisien. II a travaillé sur les effets du vaccin de l'hépatite B et a publié en 200I, La santé publique en otage (Paris, A. Michel) ; un ouvrage dans lequel il revient sur les caractéristiques du discours journalistique sur la santé en France (entretien réalisé le 31 mars 2006).
} 
Élément quelconque du milieu familier, la télévision joue la complicité avec l'univers familial grâce à ces émissions de services. C'est en partie par sa proximité avec les préoccupations des téléspectateurs que la santé à la télévision a progressivement gagné l'ensemble des magazines de société, passant ainsi de l'univers spécialisé des émissions médicales à une mise en visibilité publique dans les émissions de société diffusées en prime time. Cependant, d'autres raisons ont encouragé cette prégnance des thématiques de santé à la télévision : l'émergence de la parole ordinaire, d'une part, et, la mise en avant du malade, de sa souffrance et de ses difficultés, d'autre part. Ce processus renvoie à des évolutions sociétales qui sont relativement bien connues pour plusieurs maladies. Quelques-unes des associations de lutte contre le sida ont développé un militantisme passionné, tourné vers le malade et ses problèmes et cela aurait contribué à l'émergence de la parole ordinaire sur les plateaux, celles des malades et des victimes. Le Téléthon pour les maladies génétiques a également exposé au plus grand nombre les difficultés quotidiennes des malades (voir Walter, 1998). Pour le cancer, la manifestation que la Ligue a organisée en 1998 sous le titre « États-Généraux » a joué le rôle de révélateur de cette parole des malades. Suivant ce mouvement, les malades du cancer vont avoir eux aussi une visibilité accrue dans les émissions de télévision de société. Visibilité renforcée par l'annonce présidentielle élevant la lutte contre le cancer au rang de chantier prioritaire. Toutefois, si les associations de malades du sida ont fait évoluer les formes de militantisme et les représentations de la maladie, les associations de lutte contre d'autres maladies n'ont pas systématiquement adopté ce fonctionnement (Barbot, 2002). D'ailleurs, Sophie Aurenche le souligne en notant que « la lutte contre le cancer n'a pas interpellé de ministres ou mis de préservatif sur un monument [une des actions phare d'Act up sur l'Obélisque de la place de la Concorde à Paris]... .». Elle ajoute : « Après l'épisode sida, ce sont les médias qui se sont imposés, qui se sont autorisés à aller voir plus de malades parce qu'on avait compris sans doute qu'ils avaient une force et qu'ils pouvaient amener quelque chose $\rangle^{13}$. II s'agit donc moins d'une modification des modalités de fonctionnement et de communication des associations que d'un changement dans les pratiques des médias eux-mêmes.

\section{La santé dans les magazines de société : la force des témoignages}

La spécificité du support télévision entraîne les émissions de société à se saisir des questions de santé et à profiter ainsi de l'émergence de la parole ordinaire, mise en évidence par de multiples travaux (Mehl 1996 ; Dayan, Katz 1992 ; Cardon, Heurtin 1999), et de l'empathie provoquée

\footnotetext{
${ }^{13}$ Entretien du 30 mars 2006.
} 
par la multiplication des témoignages de malades. Ces émissions de société ont ainsi multiplié les témoignages et les reportages s'appuyant sur ce discours émotionnel, porteur d'audience. En définitive, c'est la santé et la science dans son ensemble qui sont mises en spectacle à travers un raffinement de la mise en scène qui s'éloigne des canons traditionnels du discours d'information et de la vulgarisation scientifique. Dans ces émissions, les scientifiques et les médecins ne sont donc plus les « vedettes », ils n'y ont plus la place de l'expert et ont tendance à disparaître des émissions de société, au profit des malades. Par exemple, Jean-Luc Delarue a consacré trois émissions au cancer en quatre ans ${ }^{14}$. Sur son plateau, aucun médecin n'est présent en tant qu'expert. Tout juste deux médecins sont-ils venus en fin d'émission donner un point de vue. L'absence du monde médical est également flagrante dans les reportages. Ainsi, lors du numéro de mars 200I, intitulé « Avoir une maladie grave à 20 ans », sur les quatre reportages, aucune interview de médecin, aucune prise de parole de chirurgien, ou autre spécialiste. Seule allusion au monde médical, à deux brefs instants, la caméra nous montre la réalité du traitement. Toutefois, c'est le malade qui s'administre luimême ses injections sans aucune présence de personnel médical. En règle générale, dans ces émissions de société, le présentateur a pour tâche d'animer le plateau, de convaincre les spectateurs qu'ils doivent se montrer attentifs, et d'amener les invités à s'exprimer. Sur des thèmes sensibles, comme le cancer, les témoignages sont largement suffisants pour combler le temps d'antenne, d'autant que ceux qui parlent, emploient presque toujours les « bons » mots et recréent, à partir de leurs expériences, une spectacularisation de la maladie et des soins. Ainsi, Sophie Aurenche, tout en émettant quelques réserves sur l'émission de Jean-Luc Delarue, s'est trouvée « très émue par les témoignages du numéro de Ça se discute sur le cancer dont elle était le grand témoin $\rangle^{15}$. Qu'elles réunissent des téléspectateurs sur le plateau ou qu'elles comportent de courtes séquences de reportage, les émissions sur la santé révèlent dans le public une assez large information et, un désir fréquent de se prendre en charge. De plus, le public affiche une capacité à tirer les leçons qu'apporte une expérience personnelle de la maladie. Ainsi, dans C'est au programme, une malade témoigne : «Au début on joue les braves petits soldats et puis on est tellement infantilisé par le corps médical qu'on n'ose pas poser les questions $\gg^{16}$.

\footnotetext{
${ }^{14}$ Ça se discute, le 28 mars 200I, le 5 novembre 2003 et le 16 novembre 2005.

${ }^{15}$ Entretien du 30 mars 2006.

${ }^{16}$ C'est au programme du 2 avril 2002, 9'25" à 10'07".
} 
Les formes de médiatisation de la santé dans les grands magazines de société (Ça se discute, Zone interdite, C'est au programme, Contre courant) produisent une image de souffrance et s'appuient sur l'empathie provoquée par les témoignages. Ces programmes sont portés par des animateurs (parfois animateur-producteur), non spécialistes des questions de santé (Sophie Davant, Jean-Luc Delarue ${ }^{17}$, etc.), et des malades. Ici, il n'est pas question de médecins ou d'experts. Les émissions sont construites sur des reportages portant sur la vie quotidienne des malades et sur des témoignages poignants en plateaux. Dans ce type d'émission, le témoignage a valeur d'argument contrairement aux émissions médicales où ce sont les images quelles soient de soins, d'opérations ou de spécialistes qui illustrent le discours des journalistes et des médecins. Et ce témoignage ne peut pas être contesté ni remis en cause, le malade étant le seul à avoir vécu cette expérience : il fait donc autorité. Le témoin-malade vient exposer son expérience personnelle qui devient alors une expertise (celle de sa propre expérience que nul autre ne peut partager ni discuter) et cela suffit à faire preuve. C'est l'arrivée de ce tiers qui bouleverse la structuration traditionnelle de la santé à la télévision, en provoquant une réaction des producteurs des émissions médicales.

\section{La généralisation du témoignage : la socialisation de la santé}

Si les émissions de société semblent avoir pris le pas sur les émissions médicales en terme d'audience, une réaction s'opère qui conduit ces dernières à s'approprier des procédés jusque-là spécifiques aux émissions de société, et notamment l'utilisation du témoignage. Cette généralisation rend la distinction entre émissions médicales et émissions de société de plus en plus difficile. Pour autant, les émissions médicales sont-elles en train de disparaître du secteur audiovisuel français ? Après avoir analysé l'émergence de ces témoignages dans les émissions médicales, nous proposerons une étude comparative du rôle des témoignages dans les émissions médicales et celles de société. Ainsi établirons-nous que ces témoignages sont révélateurs de l'émergence d'une forme particulière d'émissions que nous qualifions d'émissions de santé. Ces émissions ne sont plus tout à fait spécialisées, mais pas non plus des émissions de société généralistes.

\footnotetext{
${ }^{17}$ S. Davant est animatrice sur la chaîne France 2. Elle anime notamment l'émission C'est au programme depuis 200 I. L'émission est diffusée les lundi, mardi, jeudi et vendredi à 9h30. J.-L. Delarue est animateur-producteur. II présente l'émission Ça se discute sur France 2, diffusée le mardi soir en seconde partie de soirée depuis 1994. L'émission qu'il produit l'est par l'intermédiaire de sa maison de production Réservoir Prod.
} 


\section{La généralisation des témoignages}

L'utilisation accrue des témoignages se mesure à travers l'évolution de Savoir plus santé: au départ, avec Martine Allain-Régnault et François de Closets' ${ }^{18}$, un médecin était présent sur le plateau et personne d'autre. C'est lui qui détenait le savoir et la parole, et les journalistes apparaissaient essentiellement comme des présentateurs, distribuant les tours de parole, annonçant les reportages. Puis, à la fin des années 90, l'émission présentée par Martine Allain-Régnault et Laurent Broomhead donnait l'occasion à des journalistes en plateau d'interroger un médecin. Depuis l'arrivée de Sophie Aurenche en 2004, aux côtés de Laurent Broomhead, deux médecins sont présents sur le plateau ; ils discutent et débattent entre eux, mais aussi avec des journalistes et le public, « des vrais gens qui posent des questions », précise Sophie Aurenche ${ }^{19}$. Parce que les médias sont en perpétuelle recherche d'audience, et parce que les journalistes sont à l'écoute des mouvements sociétaux, les émissions médicales se sont donc tournées vers les témoignages des « vrais gens ». Cependant, la plupart du temps, il s'agit d'un témoignage-illustration qui n'a pas valeur d'argument. II vient illustrer l'argument donné par un autre, journaliste ou médecin, et il est noyé dans les images de matériel médical. De plus, la présence plus importante des malades dans les émissions médicales semble être plus quantitative que qualitative. Les malades sont invités à participer aux émissions mais ils sont presque systématiquement relégués à un statut de figurants, venant appuyer les propos d'un spécialiste. D'autres continuent à parler pour lui : journalistes, animateurs, médecins ou associations. Ainsi, lors du Magazine de la santé du 26 avril 2003, consacré pourtant à l'expression des malades ("Quand les femmes prennent la parole »), les journalistes ont bénéficié de $26,6 \%$ de temps de parole pendant le dossier, contre $40,1 \%$ pour les experts (médecins et sociologue), et seulement 15, $8 \%$ pour les malades. Dès lors comment analyser le rôle de ces témoignages?

\section{Le témoignage, ou la santé comme question sociale}

Si le développement de la parole ordinaire à la télévision a été étudié à de nombreuses reprises, la notion de témoignage reste, elle, à approfondir. En effet, « l'émergence des témoignages dans les médias a partie liée avec une mobilisation d'acteurs individuels et collectifs d'origine hétérogène [et] on ne dispose pas d'une théorisation de ce processus qui intégrerait pleinement la dimension médiatique » (Walter,

\footnotetext{
${ }^{18} \mathrm{M}$. Allain Régnault est journaliste médical, présentatrice et productrice de l'émission Savoir plus santé de 1992 à 2004 ; Fr. de Closets est journaliste à France Télévisions. Présentateur de Savoir plus santé de 1992 à 2000, date à laquelle il fut remplacé par L. Broomhead, animateur sur France 2, qui devient également co-producteur de l'émission.

${ }^{19}$ Entretien du 30 mars 2006.
} 
2005 : 8). Les témoignages de malades à la télévision posent un problème sémantique particulier : ils constituent à la fois un acte de langage, une prise de parole publique, et une illustration par l'image (celle de la personne se racontant) d'une ligne éditoriale plus large (celle de l'émission où le témoin s'exprime). Par ailleurs, ce témoignage des malades est une parole ordinaire non fondée sur la maîtrise de tel ou tel savoir mais sur une volonté individuelle de s'exprimer ; elle renvoie également à la notion de témoin c'est-à-dire à une personne venant faire part d'une situation dont elle a été le témoin direct; enfin, ces témoignages sont en plus des récits biographiques : c'est leur expérience personnelle de la maladie et leur combat contre celle-ci sur plusieurs années, vécue au jour le jour dont ils viennent faire part. C'est pourquoi le témoignage ne prend sens qu'intégré à une analyse dépassant le cadre énonciatif, alliant le contenu visuel aux contextes de production et de réception. Le rôle des témoignages dans les émissions médicales n'est compréhensible qu'en prenant en compte, d'une part, l'évolution du discours sociétal sur la santé et, d'autre part, les lignes éditoriales des différentes émissions.

Les interventions télévisées des téléspectateurs ne sont induites ni par le hasard ni par le rituel des émissions de plateau. L'assurance dont ils font preuve et les doutes qu'ils manifestent sont liés à un profond bouleversement dans l'appréhension sociale des problèmes médicaux. En parlant de la maladie, les téléspectateurs ne se bornent pas à raconter des expériences individuelles : ils traduisent également un souci collectif, ils rendent manifestes les curiosités et les craintes qui parcourent l'ensemble de la société, c'est-à-dire qu'ils conjuguent, dans leurs témoignages, ce qu'ils ont observé chez eux, dans leur milieu privé, et ce qui, constituant une préoccupation générale, relève de l'espace public. Sans doute le problème de la santé est-il très particulier car il amplifie la part de l'investissement personnel dans l'écoute télévisuelle. En parlant de leur corps, les téléspectateurs expriment à la fois un point de vue individuel et une préoccupation collective. Ce qui importe est moins l'évolution psychologique des individus que le contexte dans lequel se développe la communication médicale: « l'universalisation du particulier » (Chartier, 1990) est consacrée par le témoignage télévisuel.

Du côté de la télévision, les émissions médicales se sont données pour objectif d'intégrer la parole des malades à leur dispositif. II s'agit de se doter à la fois des caractéristiques de l'information et des spécificités de la vulgarisation scientifique. C'est pourquoi la science et le scientifique sont mis en avant: neuf personnes du corps médical sont ainsi à l'antenne lors de l'émission du 4 septembre 2004. Ces émissions diffusent une image positive de la recherche scientifique avec, par exemple, des titres d'émissions tels que « Cancer : nouvelles victoires ». 
Les témoignages s'alignent sur cette ligne éditoriale résolument optimiste : « J'ai été extrêmement bien pris en charge et je me suis retrouvé dans un centre ultra performant $\gg^{20}$. Les malades viennent témoigner de leur guérison, de leur prise en charge à temps et de leur confiance en la médecine. Contraste singulier avec les témoignages des émissions de société mettant la douleur et l'échec en avant.

Dans ces dernières, le ton est plus « polémique ». Le malade qui s'exprime sur le plateau peut mettre en doute les capacités des généralistes, souligner leurs hésitations et leurs erreurs, avec l'appui bienveillant de l'animateur. Ainsi, dans C'est au programme, Sophie Davant coupe-t-elle la parole à un médecin et déclare-t-elle : « Vous dites qu'il ne veut pas entendre la vérité, ce n'est pas vrai...Vous [montre un témoin sur le plateau], vous avez dit tout à l'heure que vous souhaitiez entendre la vérité... ». Certains n'hésitent pas à témoigner de leur décision d'abandonner leur traitement comme ce fut le cas dans chacun des trois numéros de Ça se discute consacrés au cancer. Ce statut du témoignage découle là aussi des caractéristiques de ces programmes : son utilisation pour créer de l'empathie. Les témoins y sont soigneusement choisis. Par exemple, le plateau de Ça se discute se compose des témoins représentant toutes les situations possibles sur un sujet donné. L'émission du 16 novembre 2005 présente un enfant de 5 ans en train de se battre contre la maladie, une jeune fille mannequin qui a été malade et qui s'en est sortie, un homme de 42 ans qui a vécu trois rechutes en 15 ans et qui n'ose pas s'afficher en rémission, une femme qui a accompagné son mari jusqu'à la mort, et enfin une femme de 50 ans qui a eu un cancer du sein et qui est déprimée depuis. Pour les émissions de société, il s'agit de mettre la médecine en débat et non de l'expliquer. Le discours est plus critique, les hiérarchies remises en question, les experts écartés. Et donc le témoignage y est totalement différent : il a ici valeur d'argument dans la ligne éditoriale de l'émission. II n'est plus une simple illustration, c'est sur ce témoignage que repose le message produit par l'émission. Ce n'est plus la preuve par l'image mais par le témoignage, sans remise en question ou en discussion sur le fond de ceux-ci.

La généralisation des témoignages est donc effective. En outre, d'autres procédés se sont généralisés comme l'utilisation de moniteurs en arrière-plan, ou encore la multiplication des « micro-trottoirs ». Les émissions médicales se rapprochent ainsi de plus en plus des émissions de société par l'intégration des témoignages, alors que les émissions de société n'ont jamais été aussi proches des premières en parlant de plus en plus de santé : l'émission de santé est donc bien un genre émergeant,

\footnotetext{
${ }^{20}$ Savoir plus santé le 4 septembre 2004, témoignage d'un malade.
} 
qui transcende les oppositions traditionnelles. Elle est marquée par l'ouverture de la sphère médicale à un acteur nouveau, le malade, par l'effacement relatif des scientifiques et des experts, et l'utilisation du témoignage en plateau. En effet, la santé est devenue un problème collectif dépassant le seul cadre des spécialistes. Bien plus, un espace social de la santé est en cours de construction : moins soumis aux règles purement scientifiques et plus ouverts sur les conséquences sociales de la maladie. Nous pouvons synthétiser les caractéristiques de ces émissions dans le tableau suivant :

\begin{tabular}{llllll}
\hline & Acteurs & Ligne éditoriale & Ton & $\begin{array}{l}\text { Image de la } \\
\text { science }\end{array}$ & $\begin{array}{l}\text { Valeur } \\
\text { témoignage }\end{array}$ \\
\hline $\begin{array}{l}\text { Émissions } \\
\text { médicales }\end{array}$ & $\begin{array}{l}\text { Médecins } \\
\text { Scientifiques } \\
\text { Journalistes }\end{array}$ & $\begin{array}{l}\text { Informative } \\
\text { Scientifique }\end{array}$ & Consensuel & $\begin{array}{l}\text { Positive } \\
\text { Optimiste }\end{array}$ & Néant \\
\hline $\begin{array}{l}\text { Émissions de } \\
\text { société }\end{array}$ & $\begin{array}{l}\text { Malades } \\
\text { Association } \\
\text { Animateurs }\end{array}$ & $\begin{array}{l}\text { Empathie } \\
\text { Divertissement }\end{array}$ & $\begin{array}{l}\text { Compassionnel } \\
\text { Polémique }\end{array}$ & $\begin{array}{l}\text { Négative } \\
\text { Les échecs de la } \\
\text { médecine }\end{array}$ & Argument \\
\hline $\begin{array}{l}\text { Émissions de } \\
\text { santé }\end{array}$ & $\begin{array}{l}\text { Médecins } \\
\text { Malades } \\
\text { Journalistes }\end{array}$ & $\begin{array}{l}\text { Informative } \\
\text { Empathie }\end{array}$ & Critique & $\begin{array}{l}\text { Au travail pour } \\
\text { des malades en } \\
\text { souffrance } \\
\text { Nécessité } \\
\text { d'aboutir }\end{array}$ & Illustration \\
\hline
\end{tabular}

Nous l'avons vu, ces témoignages n'ont pas le même statut : témoignageillustration dans les émissions médicales, il devient témoignage-argument dans les émissions de société, et ce en raison du contexte de production et des lignes éditoriales des ces émissions. En somme, un double discours sur la santé coexiste. D'un monde médical aux mains des spécialistes, la télévision diffuse l'image d'une médecine moins sûre d'elle, plus à l'écoute des malades. Plus tout à fait scientifiques, pas totalement émissions de société, les émissions que nous appelons de santé sont en train d'inventer un nouveau type de programme, ce qui ne va pas sans créer quelques tensions.

\section{L'autonomisation de la santé au cœur des enjeux}

Ici, la télévision est un révélateur de ce glissement du positionnement de la santé dans l'espace public. II faut élargir l'analyse pour en saisir tout le sens. Jusque vers le milieu du $X X^{\circledR}$ siècle, souffrir était une disgrâce privée dont on ne parlait qu'avec la plus grande discrétion, qu'on cachait même comme une sorte de faiblesse (Laplantine, 1986). L'acte médical était encore un tête-à-tête entre le praticien et son patient. Le changement majeur réside en ceci que la santé, autrefois souci individuel, est devenue 
un problème collectif. Affaire désormais publique, le débat sanitaire est ouvert, de la même manière que pour les transports, la famille ou l'éducation. Les médias sont contraints de lui accorder une place grandissante parce que les interventions politiques se multiplient dans ce domaine et parce que les patients, leur entourage, et la société désirent être informés et participer aux débats.

\section{La santé médiatisée au centre de débats politiques, scientifiques et médiatiques}

Si cette légère inflexion des caractéristiques des émissions médicales paraît anodine, elle révèle en fait des enjeux multiples pour le positionnement de la santé dans l'espace public: la légitimité à s'exprimer sur des thématiques de santé évolue, et ces mutations révèlent plusieurs tensions. Au niveau politique, des enjeux de société sont latents autour de cette légitimité de parole dans l'espace public, de l'émergence de nouveaux thèmes sociaux liés à la maladie (perte d'emploi, difficulté de prêt) et de la constitution d'un espace public de débat sur la santé. Les émissions de télévision accompagnent un mouvement sociétal qui les dépasse. Les citoyens ne peuvent plus être considérés par les différents pouvoirs - publics, industriels ou politique comme cet individu passif devant les choix de société qui lui sont proposés, que cela concerne l'environnement, la science ou la santé. Cette acuité des citoyens vis-à-vis des sujets de santé s'est vue renforcée par de multiples événements apparaissant comme des scandales (Marchetti, 1997 ; Henry, 2000 ; Barbot, 2002 ; Dodier, 2003) : les scientifiques et les experts mais aussi les politiques ont été remis en cause. L'intervention du Président de la République désignant le cancer comme chantier prioritaire indique également une réaffirmation des questions de santé publique comme relevant de ses prérogatives : « C'est un choix d'affirmation politique de la place de la santé publique dans la vie politique au sens large $\gg^{21}$. Après les mises en cause de la responsabilité des hommes politiques (sang contaminé, amiante, canicule,...), nous assistons à une reprise en main des affaires médicales par la sphère politique, et cette dernière le fait savoir et le montre. Ainsi Jean-François Mattei, outre 100 Minutes pour convaincre - un rendez-vous politique - a-t-il également bénéficié d'un Magazine de la santé spécial pour expliquer sa politique. La maladie met en jeu des rapports de pouvoir qu'elle exprime dans les corps à travers les différences face aux risques de l'existence ou aux possibilités de se soigner, qui sont autant de façons d'inscrire physiquement l'ordre social. Elle les révèle dans

${ }^{21}$ P. Benkimoun, journaliste spécialisé dans les questions de santé au quotidien Le Monde (entretien réalisé le ler avril 2006). 
l'intervention de ceux que l'on crédite de la capacité de guérir, mais aussi dans les relations qui s'instaurent entre les professions de santé et les pouvoirs publics. Elle les dévoile enfin dans la recherche de réponses collectives dont l'efficacité représente toujours un test pour l'autorité (Fassin 2000).

Au niveau de la recherche ensuite, la maîtrise de l'information médicale est aussi source d'enjeux pour la sphère médicale avec le partage des rôles, mais aussi de la visibilité et des fonds entre chercheurs et médecins praticiens. Les enjeux économiques entourant les grands laboratoires et les découvertes susceptibles de donner lieu à des brevets, rendent la maîtrise de l'information médicale et de sa médiatisation tout à fait essentielle pour bon nombre de centres de recherche. La maitrise de l'information médicale permet ensuite une reprise en main de la recherche sur les malades. Ces derniers avaient atteint une grande visibilité via les associations de lutte contre le sida ou après les États Généraux de la Ligue en 1998. Une place telle que le Président de la République les plaçait « au centre des préoccupations $»^{22}$. La plus ou moins grande prise en compte des malades et de leur parole finit d'ailleurs par scinder les associations entre celles tournant leurs efforts vers l'aide à la recherche, et celles se consacrant désormais aux malades et aux aspects sociaux de la maladie. La médiatisation a eu tendance à se focaliser sur ces dernières associations en délaissant les plus traditionnelles, celles tournées vers l'aide à la recherche qui apparaissent subitement « vieillottes » et décalées de l'aspiration sociale comme du chantier prioritaire. Dans ce cadre, le plan cancer et la création de I'Institut national du cancer seraient une tentative de reprise en main des chercheurs. II s'agit de fédérer les efforts pour mieux les canaliser. D'ailleurs, Jean-François Mattéi déclare : « En matière de santé publique, il faut savoir recentraliser. C'est l'État qui doit décider de ce qu'il convient de faire et les opérateurs sur le terrain le feront $\gg^{23}$.

Au niveau du média télévision, la recherche d'audience met en concurrence les émissions de société avec les émissions médicales; des tensions professionnelles entre journalistes et animateurs, et entre journalistes-médecins ou spécialistes et journalistes non spécialistes voient le jour. La montée en puissance de la parole des malades a entraîné un certain leadership en matière d'audience des émissions de société, et donc de leurs animateurs (Jean-Luc Delarue, Sophie Davant...), sur les émissions médicales et leurs journalistes spécialisés (Sophie Aurenche, Jean-Daniel Flaysakier, etc.). Ces derniers ont connu

${ }^{22}$ Allocution télévisée de J. Chirac du 14 juillet 2002.

${ }^{23} 100$ Minutes pour convaincre, 22 octobre 2002, 76'02 à 76'38". 
un fort développement depuis les années 70 et se sont structurés (Marchetti, 2002) sous l'effet de la complexité des questions liées à la santé. Cependant, devenant un problème plus social que médical, la santé vit un élargissement des questions et des thématiques en débats et donc une multiplication des acteurs et des discours. L'alignement des émissions médicales sur les émissions de société peut être interprété comme une tentative des journalistes spécialisés pour affirmer leurs prérogatives sur les animateurs. II existe en effet un certain « dénigrement » des émissions de société par les journalistes spécialisés. Ainsi Sophie Aurenche déclare-t-elle ne «pas faire le même métier $»^{24}$ que Jean-Luc Delarue. Pourtant, elle n'a pas hésité à être le grand témoin d'une de ces émissions le 16 novembre 2005. Au-delà de l'indice des tensions régnant dans ce champ professionnel, cette attitude révèle peut-être aussi l'envie d'atteindre une plus grande visibilité pour la journaliste co-présentatrice de Savoir plus santé.

Le présentateur se trouve dans la situation inconfortable de ne pas pouvoir parler de qu'il a vécu et de ne pouvoir faire état d'aucune compétence clinique. Le déséquilibre est d'autant plus évident que de nombreuses personnes non liées au corps médical sont capables d'exprimer un point de vue personnel et maîtrisent suffisamment le langage médical pour élever leur intervention à un niveau général. Sauf à devenir le modérateur d'une tribune libre, le présentateur doit faire appel à des représentants légitimes du discours global sur la pathologie, c'està-dire à des médecins pour les émissions médicales et, à des témoignages de malades pour les programmes de société. Au contraire des présentateurs, les médecins affichent le souci de la nuance et le désir d'être précis. Jusqu'à la fin des années 70, les professions médicales ont joui d'une considération dont témoignent les sit-coms et feuilletons : des médecins, entourés du respect général, s'y posaient des cas de consciences et les résolvaient aux dépends, parfois, de leur carrière, telle la série Médecins de nuit. Après 1980, la fiction est devenue moins lénifiante, les patients et les infirmières se sont mis à protester, les hôpitaux sont en difficulté, des praticiens cyniques en questionnement, comme c'est le cas dans la série Urgences.

\section{Conclusion}

Plus globalement, la façon dont les médias parlent des maladies, des traitements, des dangers, reflète la perte de prestige des médecins et la prégnance de la notion de risque : de 2000 à 2005, 30 \% des

\footnotetext{
${ }^{24}$ Entretien réalisé le 30 mars 2006.
} 
articles du journal Le Monde consacrés au cancer, l'ont fait sous l'angle du risque. Si une pandémie préoccupe particulièrement l'opinion, il devient nécessaire d'en parler là où s'opère la communication collective, donc en premier lieu à la télévision. Devenues préoccupations collectives, maladies et guérisons occupent une place croissante dans l'échange quotidien, les propos tenus à leur sujet, les nouvelles qui circulent amplifient la rumeur qui les entoure et diffusent un savoir parfois rigoureux, parfois moins, qui, en tout état de cause, est une donnée fondamentale de la culture contemporaine.

La diffusion du savoir médical et la banalisation du discours sur la santé résultent de nombreux facteurs techniques, scientifiques, économiques et culturels qui ont été relayés par les médias mais se seraient sans doute aussi bien développés sans eux. Le média télévision joue son rôle dans un ensemble de transformations dont il n'est pas le moteur. Si elle ne constitue pas le tout de l'information, elle participe davantage que n'importe quel autre support à la construction de représentation de la maladie comme du « bon » état de santé. Les représentations qu'elle diffuse ne lui appartiennent cependant pas. Elle les emprunte aussi bien à la partie de son public qu'elle fait intervenir sur les plateaux qu'à l'hôpital, aux laboratoires ou aux décisions politiques de santé publique. Forts d'un savoir acquis pour une part grâce à l'école, la presse, le commerce de la santé mais aussi dans leur milieu domestique, les spectateurs utilisent la télévision comme lieu de parole, ils viennent y confronter leur expérience à celle des autres. Face à eux, les médecins défendent leur prestige en donnant des assurances scientifiques, tout en se pliant au feu des critiques et des questions. Parce que la santé est un souci collectif et parce que les interrogations se multiplient à son sujet, le balancement entre sphère publique et sphère privée, caractéristique de la télévision, intervient de façon évidente au cours des émissions abordant les thématiques de santé.

Comprendre ce que les formes de visibilité de la santé à la télévision nous apprennent sur les mutations à l'œuvre dans ce secteur, nécessite donc de varier les niveaux d'analyse (Walter, 2005). En effet, il serait vain de chercher dans le dispositif médiatique une explication. L'étude de la parole ordinaire et des témoignages serait également insuffisante ; mais, s'en tenir à une analyse sociohistorique et sociopolitique de la santé serait tout aussi décevant. En fait, il faut accepter d'embrasser chacune de ces dimensions pour cerner la complexité des mutations en cours et leurs conséquences. Contrairement au sida, comme a pu le montrer Dominique Marchetti, le cancer n'est pas devenu un problème public : c'est un problème public ancien qui est en train d'évoluer en problème social. En effet, le cancer a toujours été au cœur de l'action et de l'attention publiques. 
Ce qui est nouveau, c'est sa « socialisation », notamment à travers les grands médias de masse. Ainsi trois niveaux d'analyse sont-ils imbriqués:

- au niveau le plus général, nous avons repéré les interactions entre plusieurs facteurs. Les dynamiques de la mise en visibilité de la santé dans les médias font appel à différentes approches historiques (science, information scientifique et sa diffusion, médias, émergence de la parole ordinaire, espace public, eux d'acteurs). C'est ainsi que nous avons retracé l'évolution de la présence de la santé à la télévision, dans notre première partie ;

- au niveau le plus particulier, le plus précis, nous nous sommes intéressée au dispositif médiatique et à l'utilisation du témoignage. Avec celui-ci, il s'agit d'apporter de la présence corporelle. C'est une façon de proposer au spectateur des points d'ancrage identificatoires qui leurs soient infiniment personnels ; ce que nous avons montré dans notre deuxième partie ;

- entre les deux, le niveau intermédiaire, le plus difficile à appréhender, les enjeux de ce positionnement de la santé et les cristallisations qui s'opèrent entre les différents acteurs font émerger les cadres interprétatifs, objet de notre troisième partie.

La médiatisation de la santé à la télévision s'intéresse dorénavant aux aspects sociaux de la maladie et non plus seulement aux aspects strictement médicaux. L'émergence d'un nouveau type d'émissions que nous avons qualifié « de santé » est révélatrice de rapports de forces importants entre plusieurs professions. Ces émissions de santé sont un compromis entre des acteurs dont une part cherche à maintenir une position dominante, une autre essaie d'accéder à ce statut et d'autres enfin qui font leur entrée dans la médiatisation de la santé. L'analyse de la santé à la télévision à travers ces trois niveaux, révèle ainsi les conflits autour de son positionnement dans l'espace public. La prégnance des questions de santé dans l'espace public et la demande d'informations ont provoqué une obligation de communiquer et donc une mise en visibilité croissante selon des modalités moins scientifiques que sociales.

\section{Références}

Barbot J., 2002, Les malades en mouvement. La médecine et la science à l'épreuve du sida, Paris, Balland.

Cardon D., Heurtin P., 1999, dirs, « Science, malades et espace public », Réseaux, 95. 
Chartier R., 1990, Les origines culturelles de la Révolution française, Paris, Éd. Le Seuil.

Dayan D., Katz E., 1992, La télévision cérémonielle. Anthropologie et histoire en direct, trad. de l'anglais par D. Dayan en collab avec J. Fendy et M. Robert, Paris, Presses universitaires de France, 1996.

Delforce B., 2004, « La médiatisation de la science : processus, dynamiques et enjeux d'une forme spécifique de publicisation », colloque « La publicisation de la science », Gresec, Grenoble.

Dodier D., 2003, Les leçons politiques de l'épidémie sida, Paris, École des hautes études en sciences sociales.

Fassin D., 2000, Les enjeux politiques de la santé, Paris, Presses universitaires de France.

Gonod M., Gubler Cl., 1996, Le grand secret, Paris, Plon.

Henry E., 2000, Un scandale improbable. Amiante : d'une maladie professionnelle à une " crise de santé publique », thèse de $3^{\mathrm{e}}$ cycle en sciences de l'homme et technologie, université de Compiègne.

Laplantine Fr., 1986, Anthropologie de la maladie. Étude ethnologique des systèmes de représentations étiologiques et thérapeutiques dans la société occidentale contemporaine, Paris, Payot.

Marchetti D., 1997, Contribution à une sociologie des transformations du champ journalistique dans les années 80 et 90. À propos d'« événements sida » et du « scandale du sang contaminé », doctorat de sociologie, École des hautes études en sciences sociales.

— 2002, « Les sous champs spécialisés du journalisme », Réseaux, I I I, vol. 20, pp. 22-55.

Mehl D., 1996, La télévision de lintimité, Paris, Éd. Le Seuil.

Miège B., 1989, La société conquise par la communication, T. I, Logiques sociales, Grenoble, Presses universitaires de Grenoble.

Pailliart I., dir., 2005, La publicisation de la science, Grenoble, Presses universitaires de Grenoble.

Romeyer H., 2004, « L'autoréflexivité télévisuelle en France. Entre communication médiatique et espace public de débat. Les cas Arrêt sur images et L'Hebdo du Médiateur », thèse en sciences de l'information et de la communication, université Stendhal-Grenoble 3.

Soulages J.-Cl., 2003, « Le traitement télévisuel de la pandémie de Sida (19852000) », communication au colloque franco-roumain Supports, dispositifs et discours médiatiques à l'heure de l'internationalisation, Bucarest 28 juin 2 juil., mise en ligne sur http://archivesic.ccsd.cnrs.fr/sic_0000078I/en/

Walter J., dir., 1998, Le Téléthon. Scène, intérêts, éthique, Paris, Éd. L'Harmattan.

- 2005, La Shoah à l'épreuve de l'image, Paris, Presses universitaires de France. 